\title{
SPACES WITH DENSE CONDITIONALLY COMPACT SUBSETS
}

\author{
ANDREW J. BERNER
}

\begin{abstract}
A subset $S$ of a topological space is said to be conditionally compact if every infinite subset of $S$ has a limit point in the space. If a space has a dense conditionally compact subset, it follows that it is pseudocompact, but the converse is not true. Examples are given of spaces that are pseudocompact, do not have dense conditionally compact subsets, but do have compactifications that are products of first countable spaces. For locally compact spaces, though, with such compactifications, the continuum hypothesis implies that pseudocompactness is equivalent to having a dense conditionally compact subset. A locally compact pseudocompact space without a dense conditionally compact subset is described.
\end{abstract}

\section{Introduction.}

Definition. A space $X$ is pseudocompact if every continuous real valued function on $X$ is bounded.

Definition. A subset $S$ of a space $X$ is conditionally compact if every infinite subset of $S$ has a limit point in $X$.

The usual, though certainly not the only, technique to prove that a topological space is pseudocompact is to show the space has a dense conditionally compact subset. Ginsburg and Saks, using a result of Frolik, proved that the type of a non- $p$ point in $\beta(N)-N$ is pseudocompact but does not contain a dense conditionally compact subset [1, Theorem 5.3]. The main result of this paper proves that (under the continuum hypothesis) a locally compact space which has a compactification which is a product of first countable spaces is pseudocompact if and only if it has a dense conditionally compact subset. Examples will be given to show that the hypothesis of local compactness cannot be removed. An example is also given of a locally compact pseudocompact space which does not have a dense conditionally compact subset. All spaces in this paper are assumed to be completely regular. Thus, pseudocompactness is equivalent to saying a space has no infinite discrete collection of open sets.

\section{Pseudocompactness and $G_{\delta}$-dense subsets.}

Definition. A subset $S$ of a space $X$ is $G_{\delta}$-dense in $X$ if every nonempty $G_{\delta}$ subset of $X$ has nonempty intersection with $S$.

It is well known, and easy to prove, that a space is pseudocompact if and only if it is $G_{\delta}$-dense in its Stone-Čech compactification. This implies that a pseudocompact space is $G_{\delta}$-dense in every compactification. It is possible, though, for a space

Received by the editors July 7, 1978 and, in revised form, February 20, 1980.

1980 Mathematics Subject Classification. Primary 54D30, 54D35; Secondary 54D45.

Key words and phrases. Pseudocompact, conditionally compact. 
to be $G_{\delta}$-dense in some compactification without being pseudocompact. For example, an uncountable discrete space is $G_{\delta}$-dense in its one point compactification. The following theorem may or may not be in the literature.

TheOREM 2.1. Suppose $X=\Pi X_{\alpha}(\alpha \in I)$ with each $X_{\alpha}$ compact and first countable, and suppose $Y$ is $G_{\delta}$-dense in $X$. Then $Y$ is pseudocompact.

Proof. Notice first of all that if $I$ is countable, then $X$ is first countable, so $Y=X$, and thus is actually compact. Suppose $\left(V_{i}\right)(i \in \omega)$ is a sequence of nonempty open sets in $Y$. Then each has a nonempty subset $U_{i} \cap Y$ where $U_{i}$ is a basic open set in $X$, thus restricted on only a finite number of coordinates $J_{i} \subset I$. Let $J=\cup J_{i}(i \in \omega)$. Since $X$ is compact, there is a point $p \in X$ such that every open set containing $p$ intersects infinitely many of the sets $U_{i}$. Since each $X_{\alpha}$ is first countable, $\{q \in X: q(j)=p(j)$ for every $j \in J\}$ is a $G_{\delta}$ in $X$. Thus there is a $p^{\prime} \in Y$ such that $p^{\prime}(j)=p(j)$ for all $j \in J$. By the definition of $J$, if $O$ is a basic open set (in $X$ ) containing $p^{\prime}$, then $O \cap U_{i} \neq \varnothing$ for infinitely many $i$ (since $O=O_{1} \cap O_{2}$ where $O_{1}$ is restricted only on coordinates in $J$, and thus contains $p$, and if $O_{1} \cap U_{i} \neq \varnothing$, then $O \cap U_{i} \neq \varnothing$ ). Suppose $V$ is an open set in $Y$ containing $p^{\prime}$. Then $V=O \cap Y$ for some open set $O \subset X$. If $O \cap U_{i} \neq \varnothing$, then $O \cap U_{i} \cap$ $Y \neq \varnothing$, i.e. $V \cap V_{i} \neq \varnothing$. Thus $V$ intersects $V_{i}$ for infinitely many $i$, showing that $Y$ is pseudocompact.

3. Some pseudocompact spaces without dense conditionally compact subsets. In this section, a class of pseudocompact spaces which do not have dense conditionally compact subsets is constructed. It will be obvious that the spaces are not locally compact, a fact which the main theorem in the next section will show is no accident.

Suppose $\kappa$ is an uncountable cardinal. The ordinal $\kappa+\omega_{1}$ has cardinality $\kappa$ and uncountable cofinality. For each $\alpha<\kappa+\omega_{1}$ suppose $X_{\alpha}$ is a first countable compact space with at least two points, and let $X=\Pi X_{\alpha}\left(\alpha<\kappa+\omega_{1}\right)$. We will construct a dense subset $Y$ of $X$ which is pseudocompact but does not have a dense conditionally compact subset.

First, define a function $f:\left[\kappa+\omega_{1}\right]^{\omega} \rightarrow \kappa+\omega_{1}$ satisfying the following condition: If $s$ is a countable subset of $\kappa+\omega_{1}$, i.e., $s \in\left[\kappa+\omega_{1}\right]^{\omega}$, then $f(s)>\sup s$. Next for each $\alpha$, pick $a(\alpha)$ and $b(\alpha)$ to be two different points of $X_{\alpha}$, and pick $U(\alpha)$ and $V(\alpha)$ to be open sets with disjoint closures containing $a(\alpha)$ and $b(\alpha)$ respectively. For each $s \in\left[\kappa+\omega_{1}\right]^{\omega}$ define

$$
Y(s)=\{p \in X: p(\alpha)=a(\alpha) \text { if } \alpha \notin s \cup\{f(s)\} \text { and } p(f(s))=b(\alpha)\} \text {. }
$$

Let $Y=\cup Y(s)\left(s \in\left[\kappa+\omega_{1}\right]^{\omega}\right)$.

Note that any nonempty $G_{\delta}$ in $X$ contains a subset that is restricted on only a countable subset $s \subset \kappa+\omega_{1}$, and thus intersects $Y(s)$. Thus $Y$ is $G_{\delta}$-dense in $X$, and therefore is pseudocompact by Theorem 2.1 .

Suppose $D$ is a dense subset of $Y$. Pick $p_{1} \in D$. Then $p_{1} \in Y\left(s_{1}\right)$ for some $s_{1}$. Let $\alpha_{1}=f\left(s_{1}\right)$. Note that $p_{1}\left(\alpha_{1}\right)=b\left(\alpha_{1}\right)$. If $p_{i}$ and $\alpha_{i}$ have been defined for $i<n$ such that $p_{i}\left(\alpha_{j}\right) \in V\left(\alpha_{j}\right)$ for $j<i$, choose $p_{n}$ such that: 


$$
p_{n} \in D, \quad p_{n}\left(\alpha_{i}\right) \in V\left(\alpha_{i}\right) \text { for } i<n, \quad p_{n}\left(\alpha_{n-1}+1\right) \in V\left(\alpha_{n-1}+1\right) .
$$

This can be done since $D$ is dense in $Y$, thus in $X$. For some $s_{n} \in\left[k+\omega_{1}\right]^{\omega}$, $p_{n} \in Y\left(s_{n}\right)$, and since $p_{n}\left(\alpha_{n-1}+1\right)$ is not $a\left(\alpha_{n-1}+1\right), f\left(s_{n}\right)>\alpha_{n-1}$. Let $\alpha_{n}=f\left(s_{n}\right)$. Therefore $p_{n}\left(\alpha_{n}\right)=b\left(\alpha_{n}\right)$. Thus the sequences $\left(p_{i}\right)(i \in \omega)$ and $\alpha_{i}(i \in \omega)$ are defined such that $p_{i} \in D$ and $p_{i}\left(\alpha_{j}\right) \in V\left(\alpha_{j}\right)$ for $j<i<\omega$. Could the sequence $\left(p_{i}\right)$ have a limit point in $Y$ ? No, since if $p$ were a limit point of $\left(p_{i}\right)$, then $p\left(\alpha_{n}\right)$ must be in $\operatorname{cl}\left(V\left(\alpha_{n}\right)\right)$ for all $n$. But then if $p \in Y(s), \alpha_{n} \in s$ for all $n$; thus $f(s)>\sup \left(\alpha_{n}\right)$, and so $p(f(s))=b(f(s))$ but $p_{n}(f(s))=a(f(s))$ for all $n$. Thus $D$ is not conditionally compact, showing that $Y$ does not have a dense conditionally compact subset.

Note. The original proof of this theorem required the hypothesis that $\kappa^{\omega}=\kappa$, and the domain of the function $f$ was $\kappa^{\omega}$. The author is grateful to Brian Scott for the clever idea of using $\kappa+\omega_{1}$ as the index set.

4. Some pseudocompact spaces which have dense conditionally compact subset. The spaces constructed in the last section were dense in a product of first countable compact spaces. It is a good thing they were not locally compact.

THEOREM $4.1(\mathrm{CH})$. If $X=\Pi X_{\alpha}(\alpha \in I)$ with each $X_{\alpha}$ compact first countable, and $Y$ is a dense pseudocompact locally compact subspace of $X$, then $Y$ has a dense conditionaly compact subspace.

Proof. As noted in the statement, we are assuming the continuum hypothesis. If $I$ is countable, then $X$ is first countable so $X=Y$. Thus we can assume $|I|>c$.

By a theorem of Arhangelskii [2, Theorem 2.21], each space $X_{\alpha}$ has a basis $\mathscr{B}_{\alpha}$ of cardinality $\leqslant c$. Thus we have a basis $\mathscr{B}$ for $X$ consisting of sets $O=\Pi O_{\alpha}$ where $O_{\alpha}=X_{\alpha}$ for all but finitely many values of $\alpha$ and for those finitely many, $O_{\alpha} \in \mathscr{B}_{\alpha}$.

We say a set $S \subset X$ is restricted only on coordinates in $J$ (where $J \subset I$, the index set of the product) if whenever $p \in S$ and $q(\alpha)=p(\alpha)$ for every $\alpha \in J$, then $q \in S$.

Note that for any set $J \subset I$ with $|J|<c$, it is true that $\mid\{B \in \mathscr{B}: B$ is restricted only on coordinates in $J\} \mid \leqslant c$.

Let $\mathscr{B}^{\prime}=\{B \in \mathscr{B}$ : closure (in $X$ ) of $B \subset Y\}$. Since $Y$ is locally compact and dense in $X, \mathscr{B}^{\prime}$ forms a basis for $Y$. Since $Y$ is open in $X$ (again because $Y$ is locally compact), $\mathscr{B}^{\prime}$ also forms a $\pi$-base for $X$.

Definition. If $J \subset I$, we say that $J$ is a good set if whenever $O$ is a basic open set in $X$ restricted only on coordinates in $J$, then there is a $V \subset \bar{V} \subset O$ such that $V \in \mathscr{B}^{\prime}$ and $V$ is restricted only on coordinates in $J$. If $J \subset J^{\prime}$ and $J^{\prime}$ is good, then we say that $J^{\prime}$ is a good extension of $J$.

LEMMA. If $J \subset I$ and $|J| \leqslant c$ then $J$ has a good extension $J^{\prime}$ with $\left|J^{\prime}\right|<c$.

Proof. Let $J(0)=J$. Suppose $J(i)$ has been defined with $J(k) \subset J(i)$ for $k<i$ and $|J(i)| \leqslant c$. For each $O \in \mathscr{B}$ restricted only on coordinates in $J(i)$, pick $V \in \mathscr{B}^{\prime}$ such that $V \subset \bar{V} \subset O$. Let $J(V, O)$ be the finite set of coordinates on which $V$ is restricted. Let $J(i+1)=J_{i} \cup(\cup J(V, O))(O \in \mathscr{B}$ and is restricted 
only on coordinates in $J(i))$. Since there are at most $c$ possibilities for $O,|J(i+1)|$ $<c$. Thus we have defined $J(n)$ for each $n \in \omega$. Let $J^{\prime}=\cup J(n)(n \in \omega)$. If $O$ is restricted only on (a finite set!) coordinates in $J^{\prime}$, then $O$ is restricted only on coordinates in some $J(n)$. Thus we chose an appropriate $V \in \mathscr{B}^{\prime}$ restricted only on coordinates in $J(n+1)$ thus in $J^{\prime}$. So $J^{\prime}$ is a good extension of $J$. Since $|J(n)|<c$ for each $n,\left|J^{\prime}\right| \leqslant c$.

To return to the proof of the theorem: Index $\mathscr{B}^{\prime}$ as $\{B(\alpha): \alpha<\kappa\}$ for some $\kappa$. For each $\beta<c$ we will define (by induction) a lot of things.

(1) $T(\beta)$ will be a good extension of $\cup T(\gamma)(\gamma<\beta)$ with $|T(\beta)|<c$.

(2) For each $\alpha<\kappa, J(\alpha, \beta)$ will be a countable subset of $I$ containing $J(\alpha, \gamma)$ for all $\gamma<\beta$.

(3) For each $\alpha<\kappa, B(\alpha, \beta)$ will be a nonempty compact subset of $\cap B(\alpha, \gamma)$ $(\gamma<\beta)$, restricted only on coordinates in $J(\alpha, \beta)$.

Also, for some values of $\beta<c$, we will define $s(\beta)$ to be a sequence in type $\omega$ of elements of $\mathscr{B}^{\prime}$ and $V(\beta)$ to be an element of $\mathscr{B}^{\prime}$. The additional properties all these objects will satisfy will be apparent from the inductive definition. The construction of all these objects follows.

Partition $c$ into $c$ disjoint sets $\{c(\alpha): \alpha<c\}$ with $|c(\alpha)|=c$, for each $\alpha$. Let $T(0)$ be an arbitrary good set of cardinality $c$. For each $\alpha<\kappa$, let $B(\alpha, 0)$ be a nonempty compact set contained in $B(\alpha)$ restricted only on some countable set (the closure of an open set, if you wish) and let $J(\alpha, 0)$ be that countable set. There are at most $c$ elements of $\mathscr{B}^{\prime}$ restricted only on coordinates of $T(0)$. Index the countable sequences of such open sets as $\{s(\gamma): \gamma>0, \gamma \in c(0)\}$.

Suppose we have defined the objects in question for all $\gamma<\beta<c$. Let

$$
\hat{T}(\beta)=\cup T(\gamma) \quad(\gamma<\beta), \quad \hat{J}(\alpha, \beta)=\cup J(\alpha, \gamma) \quad(\gamma<\beta)
$$

(note $J(\alpha, \beta)$ is countable by $\mathrm{CH}$ ),

$$
\hat{B}(\alpha, \beta)=\cap B(\alpha, \gamma) \quad(\gamma<\beta) .
$$

(Note. This is nonempty, compact and restricted only on coordinates in $J(\alpha, \beta)$.)

In the course of the induction, $s(\beta)$ may or may not have been defined. If $s(\beta)$ is not defined, let:

$T(\beta)$ be a good extension of $\hat{T}(\beta)$ with $|T(\beta)|=c$

(actually, $\hat{T}(\beta)$ is already good).

$$
J(\alpha, \beta)=\hat{J}(\alpha, \beta), \quad B(\alpha, \beta)=\hat{B}(\alpha, \beta) .
$$

If $s(\beta)$ has been defined to be $\left(B\left(\alpha_{i}\right)\right)(i \in \omega)$ and $\alpha$ is not an $\alpha_{i}$ define $J(\alpha, \beta)$ and $B(\alpha, \beta)$ as above. Let $s(\beta)=\left(B\left(\alpha_{i}\right)\right)(i \in \omega)$. For each $i$, pick a point $p_{i}$ from $\hat{B}\left(\alpha_{i}, \beta\right)$. The sequence $\left(p_{i}\right)$ has a limit point $p \in X$. Let $J(\beta)=\cup \hat{J}\left(\alpha_{i}, \beta\right)$ ( $i \in \omega$ ). Since $Y$ is (pseudocompact and thus) $G_{\delta}$ dense in $X$, there is a point $q \in Y$ such that $q(\alpha)=p(\alpha)$ for all $\alpha \in J(\beta)$. Let $q_{i}$ be defined as follows:

$$
\begin{array}{ll}
q_{i}(\alpha)=p_{i}(\alpha) & \text { for } \alpha \in J(\beta), \\
q_{i}(\alpha)=q(\alpha) & \text { for } \alpha \notin J(\beta) .
\end{array}
$$


Then $q_{i} \in \hat{B}\left(\alpha_{i}, \beta\right)$ and $q$ is a limit point of $\left(q_{i}\right)$. Let $V(\beta)$ be an element of $\mathscr{B}^{\prime}$ containing $q$ and let $J(V)$ be the finite set of coordinates on which $V(\beta)$ is restricted. For infinitely many $i, q(i) \in V(\beta)$; thus $V(\beta) \cap \hat{B}\left(\alpha_{i}, \beta\right) \neq \varnothing$. If $V(\beta) \cap \hat{B}\left(\alpha_{i}, \beta\right) \neq \varnothing$, let $B\left(\alpha_{i}, \beta\right)=\overline{V(\beta)} \cap \hat{B}\left(\alpha_{i}, \beta\right)$. If $V(\beta) \cap \hat{B}\left(\alpha_{i}, \beta\right)=\varnothing$, let $B\left(\alpha_{i}, \beta\right)=\hat{B}\left(\alpha_{i}, \beta\right)$. For all $i$, let $J\left(\alpha_{i}, \beta\right)=J(\beta) \cup J(V)$. Let $T(\beta)$ be a good extension of $\hat{T}(\beta) \cup J(\beta) \cup J(V)$ with $|T(\beta)|=c$.

Finally, whether $s(\beta)$ was defined or not, index the sequences of elements of $\mathscr{B}^{\prime}$ restricted only on coordinates in $T(\beta)$ as $\{s(\gamma): \beta<\gamma$ and $\gamma \in c(\beta)\}$. This completes the inductive definitions.

For each $\alpha$, let $B(\alpha, c)=\cap B(\alpha, \beta)(\beta<c)$. Let $T=\cup T(\beta)(\beta<c)$. Notice that $T$ is a good set (proof is similar to the end of the lemma). Let $D=\cup B(\alpha, c)$ $(B(\alpha)$ is restricted only on coordinates in $T)$. Notice that $D$ is restricted only on coordinates in $T$.

Claim. $D$ is dense in $Y$ and conditionally compact.

Proof. Suppose $O \in \mathscr{B}^{\prime}$. Then $O=O_{1} \cap O_{2}$ where $O_{1}$ is an open set in $X$ restricted only on coordinates in $T$ and $O_{2}$ is an open set in $X$ restricted only on coordinates not in $T$. Since $T$ is good, there is an $\alpha$ such that $B(\alpha) \subset O_{1}$ and $B(\alpha)$ is restricted only on coordinates in $T$. Thus $B(\alpha, c) \subset O_{1}$ and since $B(\alpha, c)$ is restricted only on coordinates in $T, B(\alpha, c) \cap O_{1} \cap O_{2} \cap D \neq \varnothing$. Thus $D$ is dense.

Suppose $\left(p_{i}\right)$ is a sequence of points from $D$. For each $i$, there is an $\alpha_{i}$ such that $p_{i} \in B\left(\alpha_{i}, c\right)$ with each $B\left(\alpha_{i}\right)$ restricted only on coordinates in $T$. There is a $\beta<c$ such that each $B\left(\alpha_{i}\right)$ is restricted only on coordinates in $T(\beta)$. Thus the sequence $\left(B\left(\alpha_{i}\right)\right)=s(\gamma)$ for some $\gamma(\gamma$ will be in $c(\beta)$ with $\beta<\gamma)$. Therefore, infinitely many of the sets $B\left(\alpha_{i}, \gamma\right)$, thus infinitely many of the points $p_{i}$, are contained in the compact set $\overline{V(\gamma)} \subset Y$ and thus $\left(p_{i}\right)$ has a limit point in $Y$. So $D$ is conditionally compact.

The set $D$ is a dense conditionally compact subset of $Y$.

5. A locally compact pseudocompact space which does not have a dense conditionally compact subset. The space constructed in this section is a variant on Gilman and Jerison's space $\psi$, with open subsets of the middle third Cantor set replacing the integers. Sequences of such sets from an appropriately defined maximal collection of almost disjoint sequences will have convergent limits, guaranteeing pseudocompactness, but some sequences of points (which will not be open) will not have limit points, keeping the space from being normally pseudocompact.

We need this observation about the middle third Cantor set.

Observation. If $\left(O_{i}\right)$ is a sequence of nonempty open sets in the middle third Cantor set (hereafter referred to as ' $C$ ') then there is a subsequence $\left(O_{i(j)}\right)$ and (possibly) another sequence of open sets $\left(V_{j}\right)$ such that $V_{j} \subset O_{i(j)}$ and $j \neq k \rightarrow V_{j}$ $\cap V_{k}=\varnothing$. (Idea of the proof: From each $O_{i}$, pick a point $p_{i}$ with $p_{i} \neq p_{j}$ for $i \neq j$. Some subsequence $p_{i(j)}$ converges to a point $p \neq p_{i(n)}$ for all $n$. Then around each $p_{i(n)}$ we can choose the appropriate sets $V_{i(n)}$.)

Let $D\left(c^{+}\right)$be the discrete space of cardinality $c^{+}$and let $N=C \times D\left(c^{+}\right)$.

Suppose $(O(i) \times\{\alpha(i)\})$ and $(U(j) \times\{\beta(j)\})$ are sequences of basic open sets 
in $N$. We will say the sequences are almost disjoint iff $O(i) \times\{\alpha(i)\} \cap U(j) \times$ $\{\beta(j)\}=\varnothing$ for all but finitely many pairs $(i, j)$.

Let $S$ be a maximal collection of pairwise almost disjoint sequences $(O(i) \times$ $\{\alpha(i)\})$ of basic open sets in $N$ having the following properties:

(1) $i \neq j$ implies that $\alpha(i) \neq \alpha(j)$ and

(2) $i \neq j$ implies that $O(i) \cap O(j)=\varnothing$.

For each sequence $s \in S$, give $s$ a convergent limit point $p(s)$ where a basic open set around $p(s)$ is defined to be $\{p(s)\} \cup \cup O(i) \times\{\alpha(i)\}(i>n)$ where $n \in \omega$.

Let $L=\{p(s): s \in S\}$ and let $X=N \cup L$. $X$ is the required space, as the following claims show.

Claim -1. $X$ is Hausdorff.

Proof. Suppose $p \neq q$. The only nontrivial case needed to show $p$ and $q$ can be separated is when $p$ and $q$ are both in $L$, in which case the definition of 'almost disjoint' guarantees that $p$ and $q$ can be separated.

Claim $0 . X$ is locally compact.

Proof. This is clear, since each basic open set in $N$ is compact, and a basic open set around a point in $L$ is just the one point compactification of the union of the sets in the sequence.

Claim 1. $X$ is pseudocompact.

Proof. Suppose $\left(U_{i}\right)$ is a sequence of open sets in $X$. Then for each $i$, there is a closed and open subset $V(i)$ of $C$ and a $\beta(i)<c^{+}$such that $V(i) \times\{\beta(i)\} \subset U_{i}$. If infinitely many values of $\beta(i)$ are the same, then the sequence has a limit point in $C \times\{\beta(i)\}$. If this is not the case, then, by the observation at the beginning of the section, we can assume that $i \neq j$ implies that $V(i) \cap V(j)=\varnothing$ and $\beta(i) \neq \beta(j)$. But then there must be a sequence $s=(O(i) \times\{\alpha(i)\})$ in $S$ such that $O(i) \times$ $\{\alpha(i)\} \cap V(j) \times\{\beta(j)\}=\varnothing$ for infinitely many pairs $(i, j)$. Note that the condition on $(\alpha(i))$ and $(\beta(i))$ guarantees that each $V(j) \times\{\beta(j)\}$ meets at most one $O(i) \times\{\alpha(i)\}$. Thus every open set around $p(s)$ intersects infinitely many of the sets $V(j) \times\{\beta(j)\}$, and thus infinitely many of the sets $U_{j}$.

Claim 2. $X$ does not have a dense conditionally compact subset.

Proof. Suppose $D$ is dense in $X$. For each $\alpha$, there is a point $p(\alpha) \in C$ such that $(p(\alpha), \alpha) \in D$. But (since $|C|=c)$ there must be a sequence $(\alpha(i))$ and a point $p \in C$ such that $p(\alpha(i))=p$ for all $i \in \omega$. But then if $(O(i)) \in S$, there is at most one $i$ such that $p \in O(i)$ (or, to be precise, the projection onto $C$ of $O(i)$ ). Thus the sequence $(p(\alpha(i)), \alpha(i))$ does not have a limit point, so $D$ is not conditionally compact.

\section{REFERENCES}

1. J. Ginsburg and V. Saks, Some applications of ultrafilters in topology, Pacific J. Math. 57 (1975), 403-418.

2. I. Juhász, Cardinal functions in topology, Math. Centre Tracts, no. 34, Math. Centrum, Amsterdam, 1971.

Department of Mathematics, University of Dallas, Irving, Texas 75061 